\title{
Use of Polyurethane Material Models for Simulating Leg-Form Impact in Different Explicit Finite Element Codes
}

\author{
Joe Hassan, Peter Schuster and G. Frederick \\ Ford Motor Company
}

Copyright 1998 Society of Automotive Engineers, Inc.

\begin{abstract}
Compressible plastic foams are used throughout the interior and bumper systems of modern automobiles for safety enhancement and damage prevention. Consequently, modeling of foams has become very important for automobile engineers. To date, most work has focused on predicting foam performance up to approximately $80 \%$ compression. However, in certain cases, it is important to predict the foam under maximum compression, or 'bottoming-out.' This paper uses one such case-a thin low-density bumper foam impacted by a pedestrian leg-form at $11.1 \mathrm{~m} / \mathrm{s}$ - to investigate the 'bottoming-out' phenomenon. Multiple material models in three different explicit Finite Element Method (FEM) packages (RADIOSS, FCRASH, and LS-DYNA) were used to predict the performance. The finite element models consisted of a foam covered leg-form impacting a fixed bumper beam with a foam energy absorber. The predicted leg-form acceleration over time was then compared to the leg-form acceleration observed during a physical test.
\end{abstract}

Within the finite element models solid elements using material types such as honeycomb, advanced foam curvilinear recoverable, strain rate foam recoverable, and low density foam were evaluated as to their accuracy in simulating Confor ${ }^{\mathrm{TM}}$ foam on the pedestrian leg-form and polyurethane energy-absorbing foam on a bumper beam under extreme compression or deformation conditions. Extreme deformation which occurs after $80 \%$ compression can cause excessive hourglassing of certain types of elements. During this extreme event many solid element material types will not exhibit the correct foam behavior, consequently the results lead to an incorrect prediction. This study attempts to determine the best material type to use during this type of large deformation impact.

\section{INTRODUCTION}

The European Commission is proposing legislation aimed at reducing the severity of injuries sustained by pedestrians in the event of an impact with the front-end of a motor vehicle [1]. One aspect of this proposed legislation is reducing the pedestrian's lower limb injuries due to contact with the bumper and frontal surfaces of a vehicle, assessed using a 'pedestrian leg impact device,' or 'legform' impactor.

As the first vehicle component contacted by the leg-form impactor, the bumper system plays the most important role in the vehicle's performance. In order to understand in more detail how the bumper system affects the leg impact, a variable buck was built and tested in a designed experiment with different geometry and stiffness levels. Details of the test setup and the results of this investigation are reported in an earlier paper [2].

The next stage of this research was to correlate a legform and generic vehicle front-end CAE concept model with the test results. While in general, the CAE concept model results were found to be similar to the test results, in one case the physical leg-form acceleration was significantly higher than that predicted by the CAE model. This particular case included both a minimal bumper energy absorber package depth $(70 \mathrm{~mm})$ and a low density (95 $\mathrm{kPa}$ stress at $40 \%$ compression) polyurethane foam energy absorber. A comparison of the acceleration plot and the high-speed video revealed that the peak acceleration occurred at the time of maximum intrusion of the leg-form into the bumper foam. This was the same time as the CAE model predicted, but in the CAE model it was easy to observe that the foam had 'bottomed-out' at that time. This paper presents the results of an investigation to identify the best finite element material model for predicting the acceleration of an object impacting a foam which 'bottoms-out.' 


\section{TEST SETUP}

Pedestrian leg impact performance is assessed through the use of a 'leg-form' impactor-two steel tubular structures connected by deformable 'ligaments' and wrapped in Confor TM foam. Although the EEVC has proposed measuring tibia acceleration, knee bend angle, and knee shear displacement, no current leg-form impactor can repeatably measure shear. Because of this, the impactor used in this test series did not include any shear measurement device. The acceleration is measured by a uniaxial accelerometer-oriented in the impact direction-in the lower structure (the 'tibia') $66 \mathrm{~mm}$ below the knee joint. The knee bend angle is measured using angular transducers at the knee joint.

The test setup consisted of a Front-End Buck [2] rigidly mounted to a steel bed-plate placed in front of a Bendix Impactor. There was a carriage attached to the impactor to support the pedestrian leg-form during the initial acceleration of the cylinder. The carriage was stopped after the initial acceleration was complete, allowing the leg to travel the last $0.6 \mathrm{~m}$ to the Variable Front-End Buck in free flight at $11.1 \mathrm{~m} / \mathrm{s}$.

The CAE models are compared to the results of a single experimental impact. Because of this, we can expect some level of experimental error in the physical test results. A recent study conducted at two European laboratories predicted a lab-to-lab test variation of up to $19.4 \%$ and $+25.6 \%$ for the acceleration [3]. Since the proposed acceptance criteria for the acceleration is 150 $\mathrm{g}$, we determined that it would be sufficient to have a CAE result which would be on the same side of the acceptance criteria (by at least $25.6 \%$ ) as the test result. This would result in the analyst correctly predicting the test result.

\section{CAE CONCEPT MODEL}

The development of the CAE concept model is described in more detail in reference [2]. Three explicit finite element codes have been used in this investigation: RADIOSS ver $3.1 \mathrm{H}$ or later, LSDYNA ver 936 or later and FCRASH ver 3.2 or later. All runs were made on Cray C90 or $J 90$ computers. Attempts were made to take advantage of the full capabilities offered by each code without bias.

LEG-FORM IMPACTOR - The simplified leg-form impactor was modeled with only nine basic parts (Figure 1). These are listed in Table (1) along with the material models used in each analysis package.

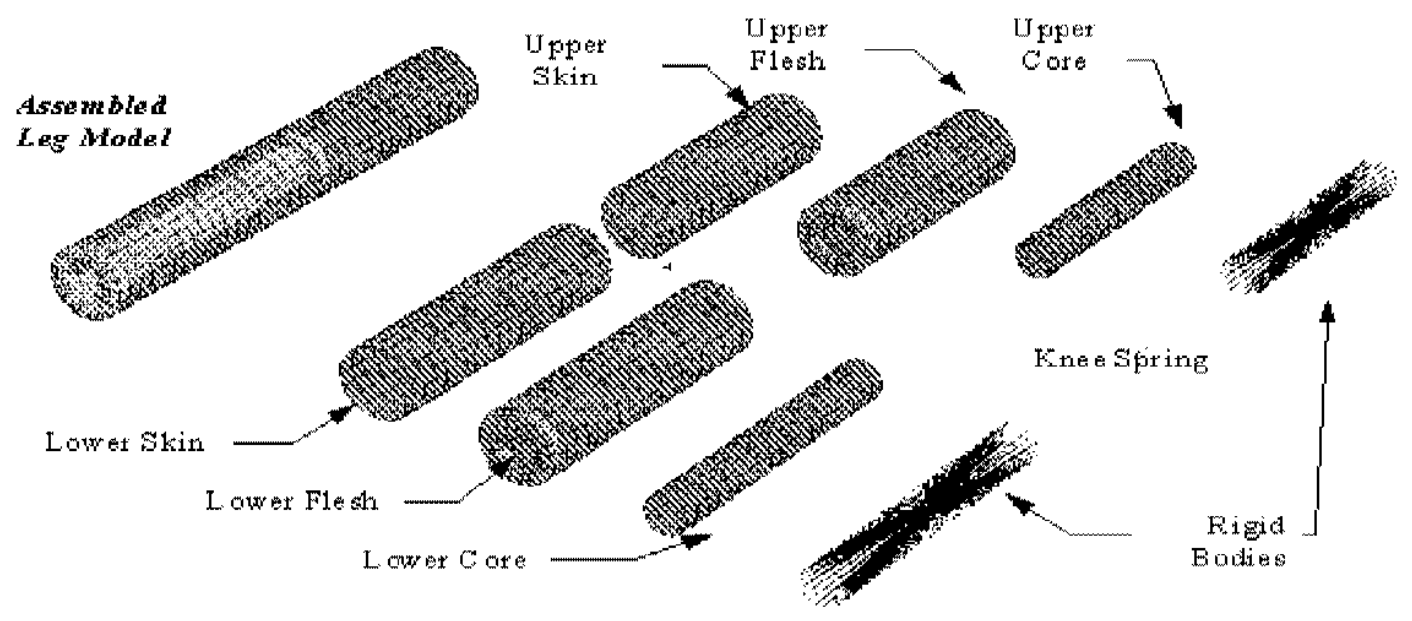

Figure 1. CAE Leg Impactor Model Construction 
Table 1. Leg-Form Impactor Material Models

\begin{tabular}{|c|c|c|c|}
\hline $\begin{array}{c}\text { Leg-Form } \\
\text { Component }\end{array}$ & $\begin{array}{c}\text { RADIOSS } \\
\text { Model }\end{array}$ & FCRASH Model & $\begin{array}{c}\text { LS-DYNA } \\
\text { Model }\end{array}$ \\
\hline $\begin{array}{c}\text { Femur and } \\
\text { Tibia Skins } \\
\text { (rubber) }\end{array}$ & $\begin{array}{c}\text { 19: Elastic } \\
\text { Orthotropic }\end{array}$ & $\begin{array}{c}\text { Linear Orthotro- } \\
\text { pic Elastic }\end{array}$ & $\begin{array}{c}\text { Orthotropic } \\
\text { Elastic Type 2 }\end{array}$ \\
\hline $\begin{array}{c}\text { Femur and } \\
\text { Tibia Flesh } \\
\text { (foam) }\end{array}$ & $\begin{array}{c}\text { 33: Low } \\
\text { Density Vis- } \\
\text { coelastic- } \\
\text { Plastic Foam }\end{array}$ & $\begin{array}{c}\text { Adv. Curvilinear } \\
\text { Recoverable } \\
\text { Foam }\end{array}$ & $\begin{array}{c}\text { Low Density } \\
\text { Foam Type 57 }\end{array}$ \\
\hline $\begin{array}{c}\text { Femur and } \\
\text { Tibia Cores }\end{array}$ & $\begin{array}{c}\text { Linear Elas- } \\
\text { tic }\end{array}$ & Linear Elastic & Elastic Type 1 \\
\hline $\begin{array}{c}\text { Femur and } \\
\text { Tibia Rigid } \\
\text { Bodies }\end{array}$ & N/A & Linear elastic & $\begin{array}{c}\text { Constrained } \\
\text { Nodal Rigid } \\
\text { Body_Inertia }\end{array}$ \\
\hline $\begin{array}{c}\text { Knee } \\
\text { Spring }\end{array}$ & $\begin{array}{c}\text { Gen. } \\
\text { Spring }\end{array}$ & General Spring & $\begin{array}{c}\text { Spring Gen- } \\
\text { eral Nonlinear }\end{array}$ \\
\hline
\end{tabular}

The model does not allow shear at the knee, in agreement with the test device. Because of this, a very simple knee model definition was applied. First, the femur and tibia segments were modeled full-length (eliminating the gap between the tibia and femur segments). Knee rotation was then allowed by specifying no interfaces between these two segments in the model. The segments were joined at the center by a zero-length general spring element.

All degrees-of-freedom for the spring element were constrained with the exception of lateral bending. For this degree-of-freedom, a non-linear function was used to define the bending properties of the knee. Isotropic hardening was used to represent the behavior of the physical knee ligaments, based on the leg impactor static bending certification corridor.

FRONT-END BUCK - Figure (2a) shows the finite element representation of the generic vehicle front-end. It includes a foam block supported rigidly at its rear face, a bumper fascia to correctly simulate the distribution of force and energy into the foam, and a lower stiffener. In addition, a grill and hood leading edge are modeled to correctly support the upper portion of the leg-form during the later stages of the impact. The initial material models used for these components are listed in Table (2). Material properties for the two foams involved in the impact are based on dynamic $(11.1 \mathrm{~m} / \mathrm{s})$ impact tests performed with sample blocks of the foams between flat steel plates.

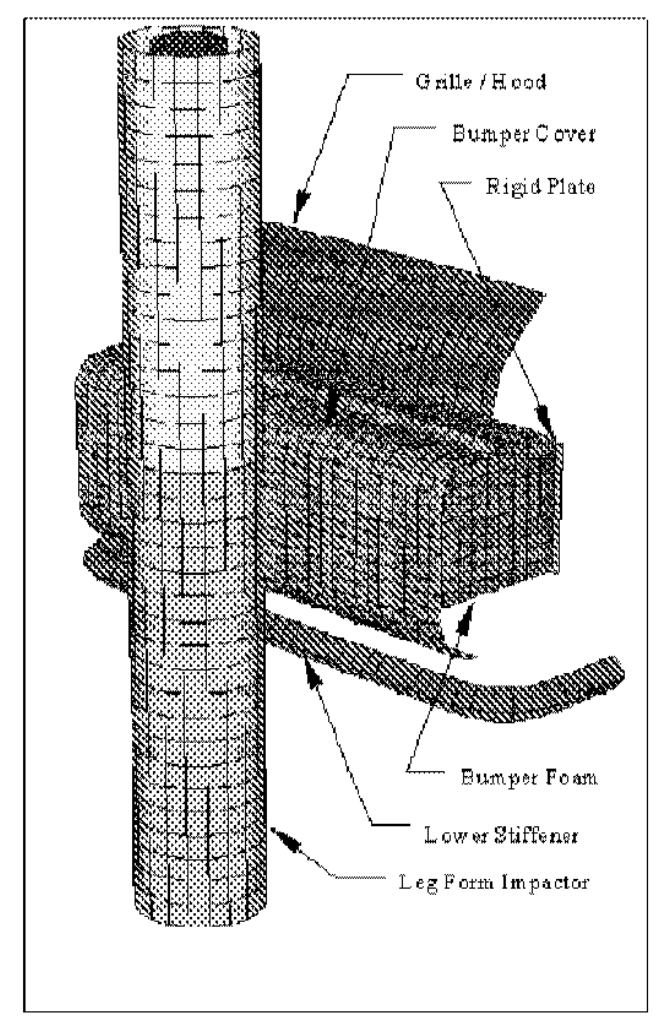

(a)

CAE Concept Model

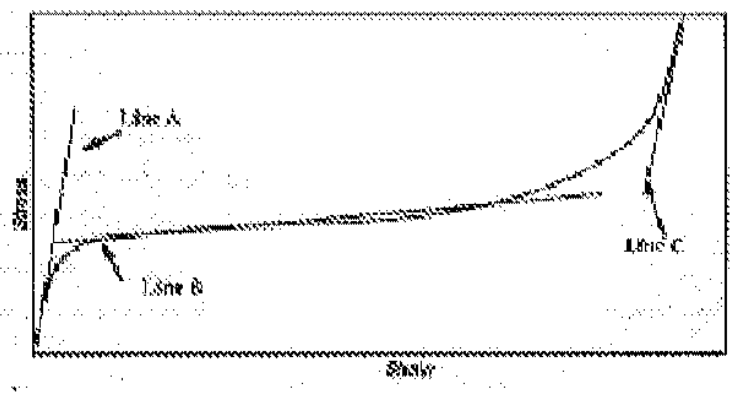

(b)

Typical Foam Response

Figure 2. CAE Concept Model and Typical Foam Response

The FCRASH model uses many master/slave (HallquistBenson algorithm) types of contact and a few self contacts (Hallquist-Benson algorithm). The RADIOSS model uses type-7 contacts while the LS-DYNA model uses automatic contacts. The models in all three codes have over 6100 nodes and 6100 elements. The material types used are linear elastic, isotropic elastoplastic, advanced foam curvilinear recoverable, and linear orthotropic elastic. The advanced foam curvilinear solid elements simulate the leg form tissue and bumper energy absorbing foam. The linear orthotropic elastic material is used to define the membrane element properties which simulate the skin of the legform. Rigid bodies are used to represent the bone structure of the legform under the tissue. 
Table 2. Front-End Buck Material Models

\begin{tabular}{|c|c|c|c|}
\hline Component & RADIOSS & FCRASH & LS-DYNA \\
\hline Bumper Fascia & $\begin{array}{l}\text { 2: Elastic } \\
\text { Plastic }\end{array}$ & $\begin{array}{c}\text { Isotropic } \\
\text { Elastoplastic }\end{array}$ & $\begin{array}{l}\text { Piecewise } \\
\text { Linear } \\
\text { Plasticity } \\
\text { (Isotropic) } \\
\text { Type } 24\end{array}$ \\
\hline Bumper Foam & $\begin{array}{l}33: \text { lowdensity } \\
\text { visco-elastic- } \\
\text { plastic foam }\end{array}$ & $\begin{array}{c}\text { Adv Foam } \\
\text { Curvilinear } \\
\text { Recoverable }\end{array}$ & $\begin{array}{l}\text { Honeycomb } \\
\text { Type } 26\end{array}$ \\
\hline $\begin{array}{l}\text { Bumper Back- } \\
\text { Plate } \\
\end{array}$ & Linear Elastic & Linear Elastic & $\begin{array}{c}\text { Linear Elas- } \\
\text { tic Type } 1 \\
\end{array}$ \\
\hline Lower Stiffener & $\begin{array}{c}\text { 2: Elastic-Plas- } \\
\text { tic }\end{array}$ & $\begin{array}{c}\text { Isotropic } \\
\text { Elastplastic }\end{array}$ & $\begin{array}{l}\text { Piecewise } \\
\text { Linear } \\
\text { Plasticity } \\
\text { (Isotropic) } \\
\text { Type } 24\end{array}$ \\
\hline $\begin{array}{l}\text { Grille/Hood } \\
\text { Leading Edge }\end{array}$ & $\begin{array}{l}\text { 2: Elastic } \\
\text { Plastic }\end{array}$ & $\begin{array}{l}\text { Isotropic } \\
\text { Elastplastic }\end{array}$ & $\begin{array}{l}\text { Piecewise } \\
\text { Limear } \\
\text { Plasticity } \\
\text { (Isotropic) } \\
\text { Type 24 }\end{array}$ \\
\hline
\end{tabular}

It is clear from the above description that the finite element model relies extensively on foam material models to describe the outcome of the analysis. To this end extensive overview of foam material modeling laws in each of the codes had to be performed for the sole purpose of taking full advantage of the capabilities offered in each of the numerical algorithms used by each code.

\section{FOAM MATERIAL SIMULATION}

Low density foam has become widely used in automobile interiors and bumper systems due to its energy absorbing capabilities at impact. It is widely known, however, that designing with foam material in such applications offers an extreme challenge to the design engineer due to its unique physical and mechanical properties. As cellular solids, foams can deform up to $90 \%$ strain in compression, while their porosity permits very large volumetric changes. This is in contrast to solid rubbers, which are approximately incompressible.

Foams are made up of polyhedral cells that pack in three dimensions. The foam cells can either be open (e.g. sponge) or closed (e.g. flotation foam). The most common use of foam materials are in cellular polymers such as cushions or padding materials which utilize the excellent energy absorption property of foams for a certain stress level.

\section{MECHANICAL BEHAVIOR OF FOAMS}

Figure (2.b) shows a typical impact test on a polypropylene foam material widely used in automotive applications. It shows a typical compressive stress-strain curve that can be defined in three distinct stages. In the first stage (strain $<5 \%$ ), the foam deforms in a mainly linear elastic manner, due to cell wall bending. The second stage can be described as a plateau of deformation at almost constant stress, caused by the elastic buckling of columns or plates, which make up the cell edges or walls.
In closed cells, the enclosed gas pressure and membrane stretching increase the level and slope of this region. The final region simulates a densification that occurs where the cell walls crush together resulting in a rapid increase of compressive stress. Ultimate compressive nominal strains of 0.7 to 0.9 are typical.

The tensile deformation mechanisms for small strains are similar to the compression mechanism but differ for large strains. At small strains for both compression and tension, the average experimentally observed Poisson's ratio of foam is $1 / 3$. At larger strains it is commonly observed that Poisson's ratio is effectively zero during compression, which indicates that the buckling of the cell walls does not result in any significant lateral deformation. However, during tension the Poisson's ratio is nonzero, which is a result of the alignment and stretching of the cell walls. Therefore, one can distinguish foam response from metals subjected to combined load cases from the mechanical behavior of each upon load application. A metal exhibits an identical volumetric stress (pressure) independently of the loading condition, where the pressure depends upon the volumetric strain only. The hydrostatic pressure in a foam however, can be 2 to 3 times higher than the uniaxial pressure at the same volumetric strain, where the pressure depends upon the volumetric strain AND upon the state-of-stress.

\section{FOAM MATERIAL MODELS IN EXPLICIT CODES}

Due to the very different behavior of foams in compression and shear, many questions remain open for interpretation due to lack of experimental evidence toward the resolution of an effective analytical treatment. Very little is known with regard to shear behavior in foams. Specifically: Does shear strength increase with compression in a combined compression/shear test? Does the longitudinal strength of a foam increase if the foam was previously compressed in the lateral direction during a biaxial or triaxial test? These questions are still unanswered in the numerical treatment of foam material laws currently available in explicit codes. However, one can identify certain specific ideas for the numerical treatment of the mechanical behavior of foams in general.

Based on the above observation one can consider foam material laws in explicit codes to be based on three main assumptions. First, a foam material can be considered a continuum with respect to its macroscopic response. Second, the foam is initially isotropic and remains isotropic during the deformation process. And third, the shear strains remain sufficiently small for the Jaumann rate to yield realistic estimates of stresses.

The above assumptions have produced 4 classes of foam material laws in numerical simulation commonly used in all explicit codes. These can be distinguished as follows:

1. Soft Polyurethanes: This specific material appears in typical applications such as seat cushions, head supports and numerous dummy parts. Generally it has 
$99 \%$ open cell structure, with density between 30 and $60 \mathrm{~g} / \mathrm{l}$, with its skin being built during forming. Initially it is isotropic and reversible. Examples of this material law is the treatment in LSDYNA type (57) and RADIOSS type (20).

2. Confor ${ }^{\mathrm{TM}}$ foam: This specific material appears in typical applications such as wheel chairs, helmets and certain dummy parts. It is generally a polyurethane foam and has open cell construction, with medium density between 60-70 g/l. It has highly damped (relaxation), reversible response with slow recovery. An example of this material law is the treatment in LSDYNA type (62) and type (33) in RADIOSS.

3. Energy-Absorbing Polyurethanes: This specific material appears in typical applications such as bumpers, knee and head bolsters and side impact padding. It has the unique characteristic that different crush strength can be obtained for a given density. It is generally made out of medium open cell construction, up to $95 \%$ closed cell, with a density between 50 and $110 \mathrm{~g} / \mathrm{l}$. It has a reversible response with slow recovery and damping. With this foam permanent deformation is possible and the plastic part of the stress-strain curve in compression is nearly horizontal. One of its most popular characteristics is that it is a good energy absorber. Irreversible E.A. Polyurethane can be simulated using material laws such as LSDYNA type $(63,53$ and 75$)$, and type (20) in RADIOSS. The reversible type of this material is usually treated as an Expanded Particle foam.

4. Expanded Particle foams: This specific material appears in typical applications such as bumpers, child seats and side impact padding. It is generally a polypropylene foam of closed cell construction $(100 \%)$, with density between 20 and $200 \mathrm{~g} / \mathrm{l}$. It has the unique characteristic that different crush strengths can be obtained for a given density. It has a reversible response with slow recovery and damping. The plastic part of stress-strain curve in compression has a definite slope. It can be distinguished by unique characteristics such as welding and the need to add holes to obtain constant force levels. Some of the applications with this material require strain rate effect. An example of this material law is the treatment in LSDYNA type (83) and type (37) in RADIOSS.

FCRASH numerical approaches treat foams based on their unloading characteristics.

Foams can be classified as hysteretic or crushable. A hysteretic foam is one that recovers its original shape when unloaded completely. The unloading path, however, deviates significantly from the loading path and as such absorbs energy. A crushable foam, on the other hand, is one that deforms permanently when compressed and does not recover its original shape when unloaded. Constitutive models are developed for both types of foam and implemented in FCRASH.
The constitutive models are developed based on the assumption that the three principal strains calculated at each time step can be used independently to compute the corresponding stresses. As such, no shear parameters-which are quite difficult to obtain experimentallyare required as user input in these models.

The input to these models are the basic stress-strain curve from a uniaxial compression test and the unloading parameters obtained from the unloading phase of the same uniaxial compression test. For a hysteretic foam model, two unloading parameters are required. These can be easily determined from the unloading response obtained from the test. For the crushable foam model, only the unloading modulus is required to characterize the unloading response. Since loading can be cyclic in nature under impact, consideration is given to the reloading event as well. Simple strain based criteria are used to distinguish the loading, unloading and reloading phases during deformation. As such, the constitutive models can effectively describe foam response under cyclic loading as well.

Foams are primarily used to withstand only compressive loading. In an actual impact however a foam may be subjected to tensile loading and experience tensile cracking or failure. To account for the tensile failure mode of foam, the constitutive models include a strain-based tension cutoff criterion. When the tension criterion is satisfied within an element at a given principal strain, the corresponding tensile principal stress is reduced to a small value as long as the tensile strain prevails. Note that the compressive behavior of the material remains unaffected by the presence of tensile failure.

Under high velocity impact, the foam may experience shock waves. Kinematic and kinetic variables such as velocity, pressure, density are generally discontinuous across such a shock wave and cause numerical problems in finite differed or finite element analyses. A shock wave smoothing technique originally proposed by von Neumann and Richtmy (1950) and subsequently modified by Landshoff (1955) is used to circumvent this problem.

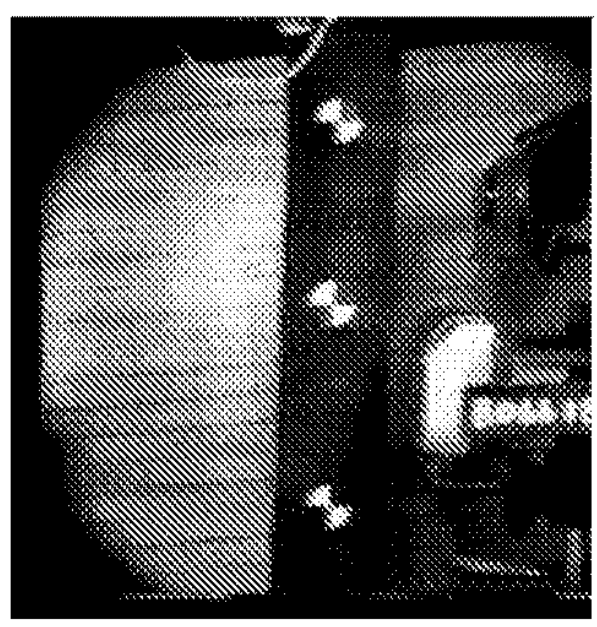

Figure 3. Test setup initial position 


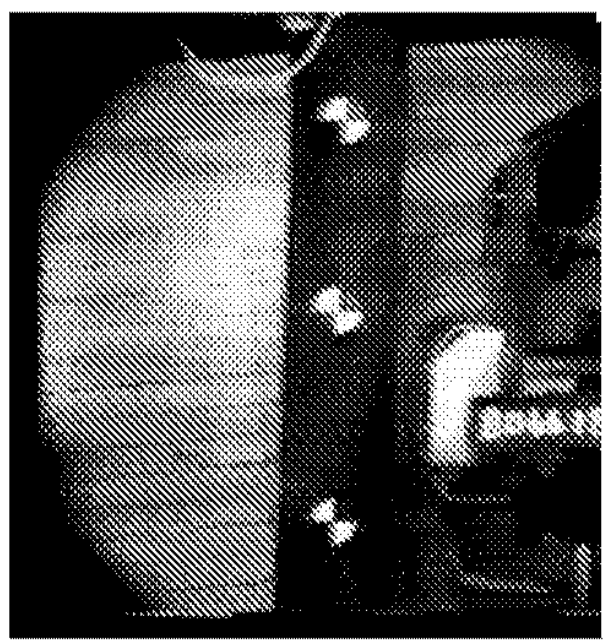

Figure 4. Test final position

\section{RESULTS AND DISCUSSION}

Comparison between results obtained from different sources such as different experiments and different test labs have to be analyzed and compared with caution due to the multitude of factors that can influence the outcome. Techniques such as repeatability and reproducibility are often relied upon to obtain some consistency in test and experimental results. The situation is no different in comparing results from different CAE codes with different foam material models.

This is due, in part to the analytical assumptions inherent in the formulation of each foam material law. Keeping that in mind while conducting this study, attention was focused on a number of parameters that can help define the response computed by each code to the same input. But control on what each model would have is simplylost from the designer due to the fact that each code handles structural components differently.

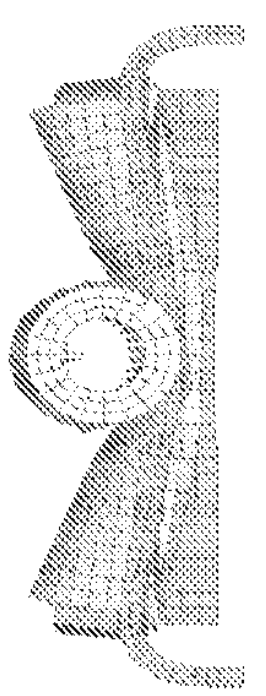

Figure 5. Typical deformation results

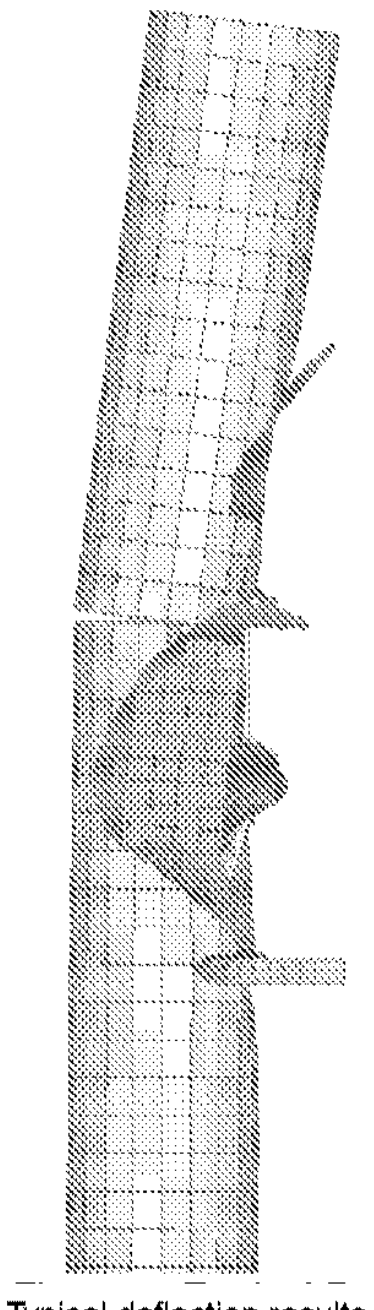

Figure 6. Typical deflection results 
Three distinct parameters were compared between the experimental test results and the analytical results obtained by all three codes. The first is the animation sequence, where animation patterns from the tests (shown in figures 3 and 4 ) were compared with animation plots from all three models. Second is bumper foam compression, where comparison was made of the penetration of the bumper foam obtained from all analytical codes at specific location points (shown in figures 5 and 6 ). The third was a comparison of the peak acceleration at the measured location using a typical impact accelerometer (as shown in figure 7).

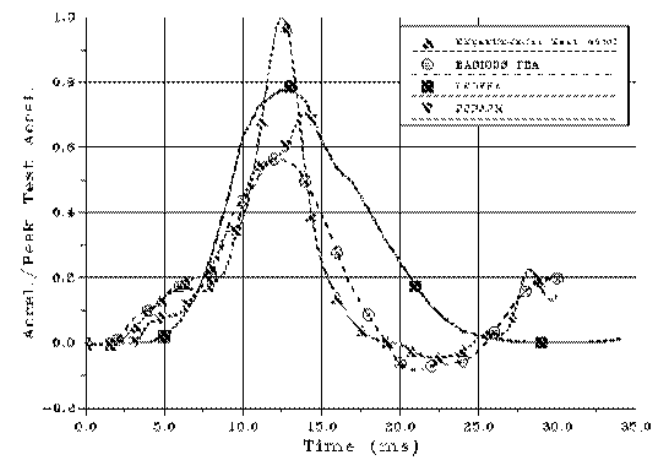

Figure 7. Tibia Normalized Acceleration

One would conclude from figure 7 that all codes have succeeded in predicting the peak behavior around the same time within a 3-5 millisecond difference. The peak value of the acceleration in the experimental results compares favorably with the analytical prediction. It can also be said that the LSDYNA model, while apparently the closest to the experimental results' peak acceleration value, also exhibits a wider response-indicating the most damping and highest model resistance. Part of this response can be attributed to the fact that the bumper foam material model used in LSDYNA was the honeycomb model, where traditionally it has inherently higher damping characteristics. FCRASH model results showed a narrower peak; however, it has less overall damping characteristics than the LSDYNA results. The RADIOSS model result was the closest to the test from the overall time response, but has the lowest peak acceleration value.

As a first attempt to understand the discrepancy between the test and finite element model results, we reviewed the test instrumentation. The uniaxial accelerometers used in the test had an off-axis sensitivity of $3 \%$. The sensitivity of the finite element acceleration measurement was $0 \%$. In order to reflect the test accelerometer sensitivity in the finite element models, the principal stress direction was identified in the vicinity of the accelerometer (by reporting principal stresses in the fascia elements). The percent of the off-axis principal stress versus the on-axis was calculated using the method described in the litera- ture [3,4]. The acceleration as reported by the CAE results was then increased by an amount equal to $3 \%$ of the acceleration resulting from the off-axis principal stresses.

\section{CONCLUSIONS}

The shape of the acceleration plot from each model closely matched the test results except around the peak acceleration point. Unfortunately, nome of the models were able to match the test peak acceleration well, using each code's defaults for the foam solid element hourglassing and foam-to-backplate interface coefficients. However, by changing the hourglass and interface coefficients to closely match the application for each code, and taking into account the off-axis sensitivity of the test accelerometer, we were able to model the peak acceleration sufficiently enough within the normal allowable engineering errors.

While it is understandable that analysts can interpet the above results as direct consequences to the foam model laws, it is however a part of an overall modeling concept and practice for each code. What affects the overall results is the fact that each code handles kinematic constraints differently. Therefore, it is wise that all aspects of the model be examined by the analyst for the sole purpose of obtaining conservative estimates of the test results. A concern that remains, however, is that it is still up to the analyst to identify when the foam in a model may have 'bottomed-out,' and anticipate some level of test acceleration or force 'spike' at that point. In the absence of correlation test results the CAE analyst needs to understand parameters that impact the characteristic behavior and not rely on default values of the CAE code to achieve conservative estimates.

\section{ACKNOWLEDGEMENTS}

The authors wish to thank Bradley Staines of Ford for his development of the leg-form impactor and front-end buck models in RADIOSS. His model formed the basis for this study.

\section{REFERENCES}

1. "Draft Proposal for the European Parliament and Council Directive Relating to the Protection of Pedestrians and Other Road Users in the Event of a Collision with a Motor Vehicle." European Commission document III/5021/96 EN, February $7,1996$.

2. Schuster, P.J. and Staines, B. "Determination of Bumper Styling and Engineering Parameters to Reduce Pedestrian Leg Injuries." SAE Paper No. 980361.

3. Green, J. "A Technical Evaluation of the EEVC Proposal on Pedestrian Protection Test Methodology." Paper No. $98-$ 510-0-04, 16th International Technical Conference on the Enhanced Safety of Vehicles, 1998.

4. J. Hassan and Machin,K.E. "transverse-Sensitivity Errors in Rectangular Rosettes"STRAIN, April, 1978.

5. J. Hassan and Machin,K.E. "Transverse-Sensitivity Errors in Strain Gage Measurements" Exp.Mec.Vol.16, No.1,pp.38-40,1976. 